\title{
Atención por teleconsulta para el personal de salud y pacientes con artritis reumatoide, en la emergencia sanitaria por covid-19: Análisis cualitativo
}

\author{
Sandra Milena Hernández Zambrano', Ruth Alexandra Castiblanco \\ Montañez ${ }^{1}$, Paula Marcela Avila Guerrero ${ }^{1}$, María Alejandra González \\ Moreno', Laura Geraldine Montenegro Sacristán ${ }^{1}$ y Pedro Santos- \\ Moreno ${ }^{2}$
}

${ }^{1}$ Fundación Universitária de Ciencias de la Salud, Colombia | smhernandez3@fucsalud.edu.co;racastiblanco@fucsalud.edu.co;pmavila@fucsalud.edu.co magonzalez5@fucsalud.edu.co;Imontenegro@fucsalud.edu.co | https://orcid.org/0000-0001 5504-3065; https://orcid.org/0000-0001-8328-9601; https://orcid.org/0000-0000-0000-0000; https://orcid.org/0000-0002-2037-9682; https://orcid.org/0000-0000-0000-0000

2Biomab -IPS, Colombia | pedrosantosmoreno@hotmail.com | https://orcid.org/0000-00017802-0317

\begin{abstract}
Resumen: Introducción La artritis reumatoide (AR) es una patología altamente vulnerable al COVID-19, factor que condujo a la implementación de la atención por teleasistencia; Objetivos; Comprender las experiencias de los pacientes con AR y los profesionales frente a la implementación de un modelo de consulta multidisciplinaria no presencial, en la emergencia sanitaria por la COVID-19. Métodos Estudio cualitativo descriptivo exploratorio. Se realizaron entrevistas semiestructuradas y se analizó siguiendo la propuesta de Taylor-Bogdan; Resultados Se realizaron 27 entrevistas, emergieron 4 categorías: Factores presentes en la comunicación, Manejo de las TIC, Apoyo e interacción familiar y Adherencia al tratamiento Conclusiones. Se identificaron condicionantes sociales, que pueden determinar la pertinencia de la teleconsulta. La implantación de la teleconsulta, debe llevar aparejada la formación de talento humano en salud, la alfabetización digital y la inversión de infraestructura tecnológica.
\end{abstract}

Palabras clave: Artritis reumatoide; Teleconsulta; COVID-19; Investigación cualitativa.

Teleconsulting care for health personnel and patients with rheumatoid arthritis, in the health emergency due to covid-19: Qualitative analysis

\begin{abstract}
Introduction Rheumatoid arthritis (RA) is a pathology highly vulnerable to COVID19, a factor that leads to the implementation of telecare assistance; Goals; Understand the experiences of RA patients and professionals in the implementation of a non-face-to-face multidisciplinary consultation model in the health emergency caused by COVID-19. Methods Qualitative descriptive exploratory study. Semi-structured interviews were carried out and it was analyzed following the Taylor-Bogdan proposal; Results 27 interviews were conducted, 4 categories emerged: Factors present in communication, ICT management, Family support and interaction, and Adherence to treatment; Conclusions. Social conditioning factors were identified, which may determine the relevance of teleconsultation. The implementation of teleconsultation must carry out the training of human talent in health, digital literacy and investment in technological infrastructure.
\end{abstract}

Keywords: Rheumatoid arthritis; Teleconsultation; COVID-19; Qualitative research.

\section{Introducción}

En el contexto de la pandemia por COVID-19 la Artritis Reumatoide (AR) es una patología vulnerable por inmunosupresión o uso de corticosteroides (Shadmanfar et al., 2020). Para evitar que la AR se exacerbe o tenga un deterioro acelerado y progresivo, se implementaron programas de teleconsulta para minimizar los riesgos de contagio de la población vulnerable y para garantizar su control y seguimiento. 
Además, el contexto de emergencia sanitaria, añade en la población cargas emocionales como la ansiedad y autoaislamiento, por miedo al contagio.

En el contexto internacional, son escasos los estudios de diseños cualitativos o mixtos que evalúan el proceso de implementación de teleconsulta en AR. En general los estudios concluyen que el modelo de la telesalud como seguimiento de la AR genera un gran impacto, ya que es una solución flexible de seguimiento a distancia, además de generar una reducción de costos (Knudsen et al., 2018); de igual forma los pacientes tienen una percepción más abierta frente a la recopilación electrónica e intercambio de información para el seguimiento, manejo y control de la AR, superando de esta manera las barreras del uso de la teleconsulta (Navarro-Millán et al., 2019).

No obstante, en el contexto colombiano no se ha identificado ningún estudio cualitativo que haya evaluado la experiencia de la teleconsulta desde la perspectiva de pacientes con AR y los profesionales de la salud.

La metodología cualitativa no mide resultados, sino que describe e interpreta un fenómeno para que se conozca de manera más compleja y profunda (Cuesta Benjumea, 2005), en este sentido, aporta evidencias contextuales para explicar las condiciones de eficacia de la intervención (López y Rivero, 2013). Por tanto, este estudio tiene como objetivo comprender las experiencias de los pacientes con AR y los profesionales frente a la implementación de un modelo de consulta multidisciplinaria no presencial, a través de llamada telefónico o video llamada, en la emergencia sanitaria por COVID-19.

\section{Metodología}

\section{- Diseño de la investigación}

Estudio cualitativo descriptivo exploratorio.

\section{- Lugar de estudio}

El estudio se realizó en la Biomab IPS (Centro de Atención Integral en Artritis Reumatoide) localizada en Bogotá, Colombia.

\section{- Población de estudio}

Pacientes adultos con AR que fueron atendidos en modalidad de teleconsulta y profesionales de la salud que laboran en Biomab IPS. Criterios de inclusión: Pacientes adultos con AR, atendidos en la modalidad de teleconsulta en al menos dos ocasiones, y profesionales de la salud que han realizado al menos 25 teleconsultas, en el contexto de la emergencia sanitaria por COVID-19.

\section{- Recolección de datos}

Se realizaron entrevistas semiestructuradas por medio de videollamada y/o llamada telefónica por la situación mundial en salud que se vive por COVID-19. La información se recolectó en un periodo de 5 semanas comprendido entre septiembre y octubre de 2020. Se utilizó un guion temático semiestructurado. Todas las entrevistas fueron grabadas en audio, con previo consentimiento informado. Se realizaron 20 entrevistas de pacientes y 7 entrevistas de profesionales para un total de 27 entrevistas. El número de entrevistas se determinó por el criterio de saturación de los datos.

\section{- Análisis de datos}

Para esta investigación se tomó la propuesta analítica de Taylor-Bogdan (1990), que responde a las preguntas de qué está sucediendo y cómo, para proporcionar una imagen de lo que la gente dice. Se realizó análisis de contenido siguiendo las etapas: preparación de los datos, descubrimiento de temas, codificación y relativización de los datos (Amezcua y Gálvez, 2002). La codificación de los datos organizó en matrices analíticas para la identificación de temas y patrones, el análisis fue en progreso, siguiendo el proceso de comparación constante e incorporación progresiva de datos hasta lograr la saturación.

$$
\text { - } 812 \text { - }
$$


Los datos de cada entrevista fueron analizados por pares y posteriormente en presencia de una tercera investigadora se realizó una triangulación global de los resultados de las 27 entrevistas, valorando criterios de confirmabilidad, credibilidad y consistencia de los datos para garantizar la validez y confiabilidad de la investigación.

\section{- Consideraciones éticas}

Este estudio fue aprobado por el Comité de Ética en Investigación con Seres Humanos HSJ-FUCS (CEISH) 0235 - 2019 del Hospital de San José. Se solicitó el consentimiento informado verbal telefónico de los participantes, garantizando el anonimato y la confidencialidad de la información obtenida.

\section{Resultados y Discusión}

Se realizaron 27 entrevistas semiestructuradas, 20 corresponden a pacientes con AR, atendidos por teleconsulta y y 7 entrevistas corresponde a los profesionales de la salud. En relación al perfil sociodemográfico, el género más prevalente fue el feminino en pacientes con \# casos $(80 \%)$ y en profesionales con \# casos $(57,14 \%)$, con un rango de edad para pacientes de $45-65$ años (58,62\%) y en profesionales de $36-50(57,14 \%)$. Respecto al perfil profesional del equipo sanitário, prevalece el médico general con $42,86 \%$, seguido de reumatólogo con $28.57 \%$, médico internista $14,29 \%$, fisiatra con $14,29 \%$ y psicólogo con $14,29 \%$.

Teniendo en cuenta la propuesta de Taylor y Bogdan (1990) (según lo descrito en la metodología) emergieron 4 categorías:

- Factores que se presentan en la comunicación y relación terapéutica que favorecen o limitan el proceso de atención

Esta categoría recoge elementos relacionados con la comunicación y relación terapéutica que influye en el proceso de la atención en salud de los pacientes con AR. La comunicación es parte fundamental de la atención sanitaria ya que garantiza la comprensión de la información entre profesional y paciente, es el momento de clarificación de dudas, además de ser un proceso de interrelación que facilita la identificación de las necesidades de cada paciente para propender por una atención personalizada.

Habitualmente la comunicación terapéutica está mediada por lenguaje corporal y verbal, no obstante, la modalidad de teleconsulta a través de llamada telefónica, limita el contacto físico y visual, hallazgo que coincide con otros estudios que evidencian la importancia del examen físico del paciente ya que por medio de este el paciente puede demostrar el empeoramiento o mejora de los signos y síntomas (Hassen et al., 2020; Tasneem et al., 2019).

Esta circunstancia conlleva a problemas en la interpretación de la información recolectada por el médico, debido a que cada paciente se expresa diferente usando términos coloquiales, o por otra parte no son muy claros al momento de comentar los síntomas. Además, la comunicación asertiva en el proceso de teleconsulta como lo refieren los médicos, se caracteriza por dar una información puntual, y clara para los pacientes siempre buscando el equilibrio a lo largo de la consulta.

\section{- Manejo de las tecnologías de la información y la comunicación (TIC)}

Esta categoría recoge los elementos relacionados con las Tecnologías de la Información y la Comunicación (TIC) que influyen en la atención de los pacientes con AR. El uso de estas TIC, intervienen en la atención en salud del paciente y permiten una comunicación terapéutica a distancia. Sin embargo, algunos participantes fueron enfáticos en las dificultades de esta modalidad de atención, destacando insatisfacción por el uso exclusivo del móvil, percepción de una atención incompleta e insatisfacción.

Algunos pacientes que cuentan con dispositivos inteligentes y conectividad a internet, sugieren el uso indispensable de la videollamada para la visualización del cuerpo del 
paciente, facilitar la valoración física, y la expresión de signos y síntomas que normalmente por una llamada no podría, y así aumentar la fiabilidad de la información clínica.

Cuando se realiza una teleconsulta en Biomab IPS se hace uso de varios dispositivos y aplicaciones de las TIC, tales como las llamadas telefónicas y celulares; el uso del aplicativo de WhatsApp o el correo electrónico.

Durante la realización de este proceso, varios de los pacientes entrevistados, han manifestado diferentes dificultades al momento de adquirir o usar estas tecnologías, lo que hace que el profesional no pueda brindar una atención adecuada y continua. La pandemia por COVID-19 ha permitido identificar falencias como equipo técnico adecuado y un nivel bajo de alfabetización, impiden un mejor aprovechamiento de estas tecnologías (Bartlett et al., 2020; Krusche et al., 2017).

Respecto a las condiciones económicas, se hace referencia al costo para la adquisición de los dispositivos de las TIC, el lugar de residencia que conlleva a la ausencia de servicio de internet y a las dificultades de recepción telefónica.

Otra dificultad percibida es la ausencia de conocimiento del manejo de las TIC, debido a que la gran mayoría de los pacientes son de la tercera edad, y para ellos hay una dificultad en el manejo de estos dispositivos. Además, desde el punto de vista de los pacientes no existe seguridad y garantía en el manejo de la información personal al momento del uso de las TIC, lo que implica riesgos en la confidencialidad de la historia clínica.

Respecto a este hallazgo, un estudio realizado en Alemania refiere que esta modalidad es complementaria, y propone optar por un modelo mixto que se adapte a las necesidades de la persona, puesto que no todas las etapas de la enfermedad son igualmente adecuadas para la utilización de la teleconsulta, y en particular con los pacientes de diagnóstico reciente (Aries et al., 2020).

- Apoyo e interacción que le ofrecen los cuidadores y/o familia a los pacientes con AR durante los diferentes procesos de atención en salud

En esta categoría se pone de manifiesto la red de apoyo de los cuidadores o la familia a los pacientes con AR durante el proceso de atención en salud, con todos los cambios que se han presentado como consecuencia de la pandemia por la COVID-19.

El rol del cuidador es asumido generalmente por un integrante de la familia que le ofrece apoyo y acompañamiento en todo el proceso de atención en salud, como, por ejemplo, la ayuda en la solución de problemas con la documentación por la virtualidad, así como la entrega de medicamentos y los diferentes exámenes solicitados por el personal médico. La asistencia y el apoyo de la familia ejercen una fuerte influencia en las percepciones de la experiencia de la AR, sobre todo en lo que respecta al bienestar psicológico, las estrategias de afrontamiento y acompañamiento en los procesos de la atención sanitaria (Bouhouche et al., 2017).

Cuando los pacientes no cuentan con esta red de apoyo, se evidenció el compromiso de la IPS, particularmente con los pacientes en edad avanzada.

\section{- Adherencia al tratamiento de los pacientes con AR}

En la AR es de vital importancia la adherencia al tratamiento para mejorar la sintomatología y percepción de bienestar. El tratamiento implica todos los seguimientos terapéuticos para llevar un control holístico, buenos hábitos de vida saludable, además del uso continuo de los medicamentos para el manejo del dolor.

Dentro de la adherencia también exponen los pacientes sus creencias religiosas sobre un ser supremo (Dios y la virgen) al que le atribuyen parte de la mejoría en su estado de salud. Sin embargo, como barrera para la adherencia se desvela el impacto de la pandemia en el acceso a los medicamentos y la atención médica, factores que reconocen otros estudios sobre enfermedades crónicas que padecen los participantes (Michaud et al., 2020). 


\section{Conclusiones}

En el contexto de la emergencia sanitaria por COVID-19, la teleconsulta como modalidad de atención desde la perspectiva de la población con AR y los profesionales, implica cambios en la relación y comunicación terapéutica entre profesionales-pacientes.

Uno de los hallazgos importantes del estudio, fue la identificación de determinantes sociales, como la alfabetización digital, la red de apoyo social, el acceso a dispositivos móviles, el acceso a internet o la recepción telefónica que pueden determinar la pertinencia de la teleconsulta, por tanto es fundamental valorar el contexto del paciente, para orientar la modalidad de atención más adecuada.

Por tanto, según los hallazgos del estudio, la implantación de la teleconsulta en el país, debe llevar aparejada la formación de talento humano en salud, la alfabetización digital y la inversión de infraestructura tecnológica en todos los rincones del país, para no perpetuar la desigualdad estructural; de no ser así, la brecha digital se convertirá en barrera de acceso y exclusión al derecho a la salud para los más desfavorecidos.

También es importante favorecer procesos de comunicación asertiva en la relación profesional-paciente mediada por TIC.

\section{Referencias}

Amezcua, M., y Gálvez Toro, A. (2002). Los modos de análisis en investigación cualitativa en salud: perspectiva crítica y reflexiones en voz alta. Rev Esp Salud Pública, 76(5), 423-36.

Aries, P., Welcker, M., Callhoff, J., Chehab, G., Krusche, M., Schneider, M., Specker, C., \& Richter, J. G. (2020). Stellungnahme der Deutschen Gesellschaft für Rheumatologie e. V. (DGRh) zur Anwendung der Videosprechstunde in der Rheumatologie [Statement of the German Society for Rheumatology (DGRh) on the use of video consultations in rheumatology]. Zeitschrift fur Rheumatologie, 79(10), 1078-1085. https://doi.org/10.1007/s00393-020-00932-x

Bartlett, S. J., De Leon, E., Orbai, A. M., Haque, U. J., Manno, R. L., Ruffing, V., Butanis, A., Duncan, T., Jones, M. R., Leong, A., Perin, J., Smith, K. C., \& Bingham, C. O. (2020). Patient-reported outcomes in RA care improve patient communication, decision-making, satisfaction and confidence: qualitative results. Rheumatology (Oxford, England), 59(7), 1662-1670. https://doi.org/10.1093/rheumatology/kez506

Bouhouche, L., Rostom, S., Idrissi, Z. M., Bahiri, R., \& Hajjaj-Hassouni, N. (2017). Perceptions in Rheumatoid Arthritis: Comparative Study Between Patients and Their Close Families. Journal of clinical rheumatology : practical reports on rheumatic \& musculoskeletal diseases, 23(5), 262-266. https://doi.org/10.1097/RHU.0000000000000478

Cuesta Benjumea, C. (2005). La contribución de la Evidencia Cualitativa al campo del cuidado y la salud comunitaria. Index Enferm, 14( 50 ), 47-52.

Hassen, L. M., Almaghlouth, I. A., Hassen, I. M., Daghestani, M. H., Almohisen, A. A., Alqurtas, E. M., Alkhalaf, A., Bedaiwi, M. K., Omair, M. A., Almogairen, S. M., Alarfaj, H. F., \& Alarfaj, A. S. (2020). Impact of COVID-19 outbreak on rheumatic patients' perceptions and behaviors: A cross-sectional study. International journal of rheumatic diseases, 23(11), 1541-1549. https://doi.org/10.1111/1756-185X.13959

Knudsen, L.R., de Thurah, A., \& Lomborg, K. (2018). Experiences With Telehealth Followup in Patients With Rheumatoid Arthritis: A Qualitative Interview Study. Arthritis Care Res, 70(9), 1366-1372.

Krusche, M., Mühlensiepen, F., Aries, P., Welcker, M., \& Knitza, J. (2020). Telemedizin in der Rheumatologie [Telemedicine in rheumatology]. Zeitschrift fur Rheumatologie, 79(9), 883-892. https://doi.org/10.1007/s00393-020-00912-1

López, S., \& Rivero, S.C. (2013). Necesidad de desnudar las evidencias enfermeras desde el Realismo Crítico. Evidentia.; 10(41). Disponible en: <http://www.indexf.com/evidentia/n41/ev4100.php> Consultado el 26 de Febrero de 2021. 
Vol. 8 | Investigação Qualitativa em Saúde: Avanços e Desafios

Michaud, K., Wipfler, K., Shaw, Y., Simon, T. A., Cornish, A., England, B. R., Ogdie, A., \& Katz, P. (2020). Experiences of Patients With Rheumatic Diseases in the United States During Early Days of the COVID-19 Pandemic. ACR open rheumatology, 2(6), 335-343. https://doi.org/10.1002/acr2.11148

Navarro-Millán, I., Zinski, A., Shurbaji, S., Johnson, B., Fraenkel, L. \& Willig, J. (2019). Perspectives of Rheumatoid Arthritis Patients on Electronic Communication and Patient-Reported Outcome Data Collection: A Qualitative Study. Arthritis Care Res, 71(1), 80-87

Shadmanfar, S., Jonaidi-Jafari, N., Jafari, R., Rastgar-Moqaddam, Z., \& Saburi A. (2020). COVID-19 in rheumatoid arthritis cases: an Iranian referral center experience. Clin Rheumatol, 20, 1-6.

Tasneem, S., Kim, A., Bagheri, A., y Lebret, J. (2019). Telemedicine Video Visits for patients receiving palliative care: A qualitative study. The American journal of hospice \& palliative care, 36(9), 789-794. https://doi.org/10.1177/1049909119846843

Taylor, S.J., y Bogdan, R. (1990). Introducción a los métodos cualitativos de investigación. Barcelona: Paidós. 